\title{
Two-component plasma in a gravitational field: Thermodynamics
}

\author{
Gabriel Téllez* \\ Laboratoire de Physique \\ Ecole Normale Supérieure de Lyon, 46 Allée d'Italie, 69364 Lyon \\ cedex 07, France
}

\begin{abstract}
We revisit the model of the two-component plasma in a gravitational field, which mimics charged colloidal suspensions. We concentrate on the computation of the grand potential of the system. Also, a special sum rule for this model is presented.
\end{abstract}

\section{INTRODUCTION}

In a recent paper, the author presented a particular solvable model of Coulomb system inspired from the problem of the sedimentation equilibrium of charged colloidal suspensions. Although the model is much simpler than real colloidal suspensions, it features several properties observed in numerical results for more realistic models 2 and shows some new interesting results.

This Communication is a complement to Ref. 1 in which only the density profiles where computed. Here we concentrate on thermodynamic quantities such as the grand potential. In section $\mathbb{I}$ we briefly describe the model, in section III we compute the grand potential of the system, and in the last section we present a special sum rule for this model.

*e-mail address: gtellez@physique.ens-lyon.fr

${ }^{\dagger}$ Laboratoire associé au Centre National de la Recherche Scientifique - URA 1325 


\section{THE MODEL}

We consider a two-dimensional two-component plasma composed of two species of particles with charges $\pm q$ and masses $M_{ \pm}$. We choose a system of Cartesian coordinates $(O x y)$ in which the gravitational field is $\mathbf{g}=-g \hat{\mathbf{y}}$. The particles are in a container of height $h$ and infinite width. Let us define the inverse gravitational lengths of the particles $k_{ \pm}=\beta M_{ \pm} g, k_{0}=k_{+}+$ $\left.k_{-}\right) / 2$, and $\delta k=k_{+}-k_{-}$, where $\beta$ is the inverse temperature. In the grand canonical ensemble we define position-dependent rescaled fugacities $m(\mathbf{r})=$ $m_{0} \exp \left(-k_{ \pm} y\right)$ to account for the interaction of the particles with the external gravitational field. The screening length (at zero altitude) is given by $1 / m_{0}$, , 目

The two-component plasma is equivalent to a free Dirac field $\mathrm{A}_{\mathrm{G}} \mathrm{G}$ when $\beta q^{2}=2$. The grand partition function can be written as $\Xi=\operatorname{det}(1+K)$, where

$$
K=\left[m_{+}(\mathbf{r}) \frac{1+\sigma_{z}}{2}+m_{-}(\mathbf{r}) \frac{1-\sigma_{z}}{2}\right] \not^{-1}
$$

with $\sigma_{x}, \sigma_{y}, \sigma_{z}$ the Pauli matrices and $\not \partial=\sigma_{x} \partial_{x}+\sigma_{y} \partial_{y}$. To compute $\Xi$, one must solve the eigenvalue problem

$$
K \Psi=\lambda \Psi
$$

where $\Psi=(\psi, \chi)$ and $\lambda$ are the eigenvectors and eigenvalues of $K$. Then the grand potential is given by

$$
\Omega=-k_{B} T \sum_{\lambda} \ln (1+\lambda)
$$

When an external field is acting differently over the positive and negative particles, as in the present case, it is useful to write the fugacities as $m_{ \pm}(\mathbf{r})=$ $m(\mathbf{r}) \exp [-( \pm) 2 V(\mathbf{r})]$ where $m(\mathbf{r})=m_{0} \exp \left[-k_{0} y\right]$ and $V(\mathbf{r})=\delta k y / 4$. Let us define the auxiliary eigenfunctions

$$
\begin{aligned}
& \phi_{+}(\mathbf{r})=e^{V(r)} \psi(\mathbf{r}), \\
& \phi_{-}(\mathbf{r})=e^{-V(r)} \chi(\mathbf{r}),
\end{aligned}
$$


and the operators

$$
a=\partial_{x}+i \partial_{y}+\partial_{x} V(\mathbf{r})+i \partial_{y} V(\mathbf{r})
$$

and

$$
a^{\dagger}=-\partial_{x}+i \partial_{y}+\partial_{x} V(\mathbf{r})-i \partial_{y} V(\mathbf{r}) .
$$

Then the eigenvalue problem $(2.2)$ is equivalent to

$$
\begin{gathered}
\phi_{+}(\mathbf{r})+\lambda^{2} a^{\dagger} m(\mathbf{r})^{-1} a\left[m(\mathbf{r})^{-1} \phi_{+}(\mathbf{r})\right]=0, \\
\phi_{-}(\mathbf{r})=\lambda a\left[m(\mathbf{r})^{-1} \phi_{+}(\mathbf{r})\right],
\end{gathered}
$$

and the boundary conditions that $\psi(\chi)$ on the boundary is equal to a function which is analytic (anti-analytic) outside the container and vanishes at infinity 6 . This method is general and can be applied to others models of two-component plasma in an external field.

\section{THE GRAND POTENTIAL}

In the present case, we look for solutions of (2.8) of the form $\phi_{+}(\mathbf{r})=$ $h(y) \exp \left(i k x-k_{0} y\right)$. From (2.8) we find the equation for $h(y)$

$$
\left[(k+\delta k / 4) k_{0}-(k+\delta k / 4)^{2}-\lambda^{-2} m_{0}^{2} e^{-2 k_{0} y}\right] h(y)+k_{0} h^{\prime}(y)+h^{\prime \prime}(y)=0,
$$

which is very similar to the equation (3.2) of Ref. 1 satisfied by the Green function $\hat{g}_{++}$. The solution is

$$
h(y)=\left[A I_{\nu}\left(\frac{m_{0} e^{-k_{0} y}}{\lambda k_{0}}\right)+B K_{\nu}\left(\frac{m_{0} e^{-k_{0} y}}{\lambda k_{0}}\right)\right] e^{-k_{0} y / 2},
$$

with $\nu=\left|k-\left(k_{-} / 2\right)\right| / k_{0}$, and $A$ and $B$ constants of integration.

In the present case, the boundary conditions become $\phi_{+}(x, y=0)=0$ and $\phi_{-}(x, y=h)=0$ if $k>0$, and $\phi_{+}(x, y=h)=0$ and $\phi_{-}(x, y=0)=0$ if $k<0$. These conditions give a system of two linear homogeneous equations

for the constants $A$ and $B$. Writing that the discriminant of this system 
must be zero, in order to have non-trivial solutions, gives the equation that determines $\lambda$. Let $\sigma$ be the sign of $k-\left(k_{-} / 2\right)$. If $k>0, \lambda$ is a solution of

$$
I_{\nu}\left(\frac{m_{0}}{\lambda k_{0}}\right) K_{\nu+\sigma}\left(\frac{m_{0} e^{-k_{0} h}}{\lambda k_{0}}\right)+I_{\nu+\sigma}\left(\frac{m_{0} e^{-k_{0} h}}{\lambda k_{0}}\right) K_{\nu}\left(\frac{m_{0}}{\lambda k_{0}}\right)=0,
$$

and if $k<0, \lambda$ is given by

$$
I_{\nu}\left(\frac{m_{0} e^{-k_{0} h}}{\lambda k_{0}}\right) K_{\nu-1}\left(\frac{m_{0}}{\lambda k_{0}}\right)+I_{\nu-1}\left(\frac{m_{0}}{\lambda k_{0}}\right) K_{\nu}\left(\frac{m_{0} e^{-k_{0} h}}{\lambda k_{0}}\right)=0 .
$$

These equations have an infinite number of solutions. For each $k$, we may index the different values of $\lambda$ by an integer $\ell: \lambda=\lambda_{k, \ell}$. Then, the grand potential by unit length in the $x$-direction is

$$
\omega=-\frac{k_{B} T}{2 \pi} \int \sum_{\ell} \ln \left(1+\lambda_{k, \ell}\right) d k .
$$

The sum over $\ell$ can be done explicitly, $k<0$, by noting that the zeros of the entire function

$$
\begin{aligned}
f(z)=\frac{m_{0}}{k_{0}} e^{-k_{0} h \nu} z[ & I_{\nu}\left(m_{0} e^{-k_{0} h} z / k_{0}\right) K_{\nu-1}\left(m_{0} z / k_{0}\right) \\
& \left.+I_{\nu-1}\left(m_{0} z / k_{0}\right) K_{\nu}\left(m_{0} e^{-k_{0} h} z / k_{0}\right)\right],
\end{aligned}
$$

are $1 / \lambda_{k, \ell}$ and that $f(0)=1$, so $f(z)=\prod_{\ell}\left(1-\lambda_{k, \ell} z\right)$. Then,

$$
\sum_{\ell} \ln \left(1+\lambda_{k, \ell}\right)=\ln \prod_{\ell}\left(1+\lambda_{k, \ell}\right)=\ln f(-1) .
$$

The same can be done in the case $k<0$. Then, the grand potential can be expressed as three integrals for $k$ in the domains $]-\infty, 0],\left[0, k_{-} / 2\right]$ and $\left[k_{-} / 2,+\infty[\right.$. After a change of variable in the integrals, the grand potential can be written in a more compact way as

$$
\begin{aligned}
\beta \omega=-\frac{k_{0}}{2 \pi}\left(\int_{-k_{-} / 2 k_{0}}^{+\infty}+\int_{-k_{+} / 2 k_{0}}^{+\infty}\right) \ln \left[e ^ { - k _ { 0 } h ( \nu + 1 ) } \frac { m _ { 0 } } { k _ { 0 } } \left\{I_{\nu}\left(\frac{m_{0}}{k_{0}}\right) K_{\nu+1}\left(\frac{m_{0}}{k_{0}} e^{-k_{0} h}\right)\right.\right. \\
\left.\left.+I_{\nu+1}\left(\frac{m_{0}}{k_{0}} e^{-k_{0} h}\right) K_{\nu}\left(\frac{m_{0}}{k_{0}}\right)\right\}\right] d \nu .
\end{aligned}
$$


From now on we consider the simpler case when the container has infinite width $(h \rightarrow \infty)$. Equation (3.8) becomes

$$
\beta \omega=-\frac{k_{0}}{2 \pi}\left(\int_{-k_{-} / 2 k_{0}}^{+\infty}+\int_{-k_{+} / 2 k_{0}}^{+\infty}\right) \ln \left[\left(\frac{2 k_{0}}{m_{0}}\right)^{\nu} \Gamma(\nu+1) I_{\nu}\left(\frac{m_{0}}{k_{0}}\right)\right] d \nu .
$$

The two-component plasma at $\beta q^{2}=2$ has logarithmic divergences due to the collapse of pairs of particles of opposite sign. This can be avoided by introducing hard core particles of radius $R$. The integrals in (3.8) and (3.9) are divergent and must be cutoff, the upper limit of integration becomes $\nu_{\max }=\left(k_{0} R\right)^{-1}$.

We can have an asymptotic expression for the grand potential in the

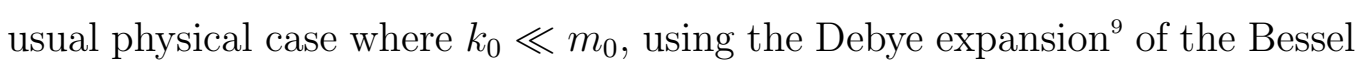
functions. The calculations are very similar to those of Ref. 6 for a different problem. One finds

$$
\omega=-\frac{1}{2 k_{0}} p_{0}+o\left(\frac{m_{0}}{k_{0}}\right),
$$

where $p_{0}$ is the pressure of a two-component plasma without an external field. It is interesting to notice that this is the same grand potential of a system without gravity, but confined in a container of height $\left(2 k_{0}\right)^{-1}$. Furthermore, from Ref. 1, we know that most of the particles are in fact in the region $0<y<\left(2 k_{0}\right)^{-1}$ since for intermediate altitudes (the neutral zone) the density profiles decay as $\exp \left(-2 k_{0} y\right)$.

\section{A SPECIAL SUM RULE}

We can write a sum rule for this system by computing in two different ways the force exerted on the bottom of the container. This force is the weight of the column of fluid over the base of the container. On the other hand, it is also given by the pressure at zero altitude which can be computed by means of the contact theorem: it is the density at zero altitude times $k_{B} T$. This gives the sum rule

$$
\rho_{+}(0)+\rho_{-}(0)=\beta g\left(M_{+} \int_{0}^{+\infty} \rho_{+}(y) d y+M_{-} \int_{0}^{+\infty} \rho_{-}(y) d y\right)
$$


where $\rho_{ \pm}$are the individual densities of the positive and negative particles respectly. This sum rule can be written in a simpler way; because the system is neutral, both integrals in (4.1) are equal to $n / 2$, where $n$ is the total number of particles. Then, the sum rule becomes

$$
\rho_{+}(0)+\rho_{-}(0)=k_{0} n \text {. }
$$

We expect this to be true at any temperature. We can test the sum rule in the present case when $\beta q^{2}=2$. The total number of particles can be computed from the grand potential:

$$
n=-\beta m_{0} \frac{\partial \omega}{\partial m_{0}} .
$$

From equation (3.9) one finds

$$
n=\frac{m_{0}}{2 \pi}\left(\int_{-k_{-} / 2 k_{0}}^{+\infty}+\int_{-k_{+} / 2 k_{0}}^{+\infty}\right) \frac{I_{\nu+1}\left(\frac{m_{0}}{k_{0}}\right)}{I_{\nu}\left(\frac{m_{0}}{k_{0}}\right)} d \nu .
$$

On the other hand, from equation (3.10) of Ref. 11 we can compute the densities at zero altitude:

$$
\rho_{ \pm}(0)=\frac{m_{0} k_{0}}{2 \pi} \int_{-k_{ \pm} / 2 k_{0}}^{+\infty} \frac{I_{\nu+1}\left(\frac{m_{0}}{k_{0}}\right)}{I_{\nu}\left(\frac{m_{0}}{k_{0}}\right)} d \nu .
$$

The sum rule (4.2) is verified.

\section{ACKNOWLEDGMENT}

I wish to thank B. Jancovici for his remarks on an early version of the manuscript.

${ }^{1}$ G. Téllez, J. Chem. Phys. 106, 8572 (1997).

${ }^{2}$ T. Biben and J. P. Hansen, J. Phys. Condensed Matter 6, A345 (1994). 
${ }^{3}$ F. Cornu and B. Jancovici, J. Chem. Phys. 90, 2444 (1989).

${ }^{4}$ S. Coleman, Phys. Rev. D 11, 2088 (1975).

${ }^{5}$ S. Samuel, Phys. Rev. D 18, 1916 (1978).

${ }^{6}$ B. Jancovici, G. Manificat, C. Pisani, J. Stat. Phys. 76, 307 (1994).

${ }^{7}$ B. Jancovici and G. Téllez, J. Stat. Phys. 82, 609 (1996).

${ }^{8}$ P. J. Forrester, J. Stat. Phys. 67, 433 (1992).

${ }^{9}$ M. Abramowitz and I. Stegun, Handbook of Mathematical Functions (National Bureau of Standards, Washington, D. C., 1964). 